\title{
Supporting Information \\ CO oxidation with atomically dispersed catalysts: Insights from the energetic span model
}

\author{
Selin Bac, ${ }^{a}$ and Shaama Mallikarjun Sharada*a,b \\ ${ }^{a}$ Mork Family Department of Chemical Engineering and Materials Science, University of Southern California, \\ Los Angeles CA, USA 90089 \\ ${ }^{b}$ Department of Chemistry, University of Southern California, Los Angeles CA, USA 90089 \\ E-mail: ssharada@usc.edu
}

\section{Methods}

Density functional theory: We perform spin polarized calculations using Vienna Ab Initio Simulation Package, VASP 5.4. ${ }^{1-4}$ Strongly-Constrained and Appropriately-Normed (SCAN) meta-generalized gradient approximation (meta-GGA) is adopted for calculations. ${ }^{5}$ The Atomic Simulation Environment (ASE) is utilized for model construction and geometry relaxation with a threshold of $0.05 \mathrm{eV} / \AA .{ }^{6}$ All atoms are described using the default projector augmented wave (PAW) potentials in which $\mathrm{Ti}, \mathrm{O}, \mathrm{Pt}$, and $\mathrm{C}$ contains four, six, ten, and four valence electrons, respectively. ${ }^{7,8} \Gamma$-point sampling with Gaussian smearing $(\sigma=0.1 \mathrm{eV})$ is used along with cutoff energy of 400 $\mathrm{eV}$. Bader analysis is employed to calculate charges of carbonate intermediate and $\mathrm{Pt}$ atoms throughout reaction cycles. ${ }^{9,10}$ Binding energies (BE) are calculated using reference energies of gas phase atoms/molecules and the surface:

$$
B E=E_{a d s-P t / T i O_{2}}-E_{P t / T i O_{2}}-E_{\text {molecule/atom,gas }}
$$

where $E_{a d s-P t / T i O_{2}}$ is the energy of the surface with adsorbed species.

Transition States: Transition states are determined using the nudged elastic band (NEB) method followed by climbing-image nudged elastic band (CI-NEB). ${ }^{11-13}$ We create at least four images between initial and final structures. The threshold of convergence is set to $0.05 \mathrm{eV} / \AA$ with $400 \mathrm{eV}$ cutoff energy. The transition states are verified by vibrational analysis, in which a single imaginary frequency is expected.

Vibrational Analysis: The Vibrations package in ASE is used for vibrational analysis calculations. ${ }^{14}$ A finite difference displacement of $0.01 \AA$ is chosen. For every structure, all atoms except are kept frozen except for Pt, its nearest neighbors and adsorbates or reaction intermediates. In each catalytic cycle, the number of atoms on the surface (except reaction intermediates) that are allowed to vibrate is kept constant.

Free Energies: The Gibbs free energies are calculated for all intermediates and transition states: ${ }^{15}$

$$
\Delta G=\Delta E_{D F T}+\Delta E_{Z P E}+\Delta U-T \Delta S
$$

where $E_{D F T}$ is the energy obtained from the DFT calculations, $E_{Z P E}$ is the zero-point energy obtained from the vibrational analysis and $\mathrm{T}$ is the temperature. Internal energy $(U)$ corresponds to vibrational enthalpy contributions excluding the zero point energies. The total entropy $(S)$ is obtained from the translational, rotational and vibrational contributions for the gas-phase molecules: ${ }^{15}$

$$
S=S_{\text {trans }}+S_{\text {rot }}+S_{\text {vib }}
$$

Translational, vibrational and rotational contributions to the free energy are calculated at reaction temperature from corresponding partition functions. ${ }^{16}$ Translations and rotations are assumed to be quenched for all bound species, with the exception of TS2 in Figure S11 for which entropy loss to adsorption is not included as $\mathrm{CO}_{2}$ is very close to a free molecule. We replace any frequency below $50 \mathrm{~cm}^{-1}$ with $50 \mathrm{~cm}^{-1}$ to minimize divergence of vibrational entropy contributions stemming from low-frequency modes. ${ }^{17}$ We note that the effect of reactant and product concentrations (partial pressures) are neglected in this work. This is because, as we show in Section 4 of the SI, inclusion of pressure contributions in $\Delta G$ and TOF calculations do not impact mechanistic conclusions. 


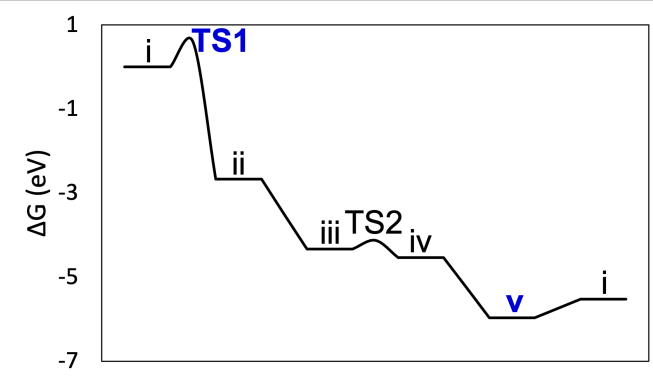

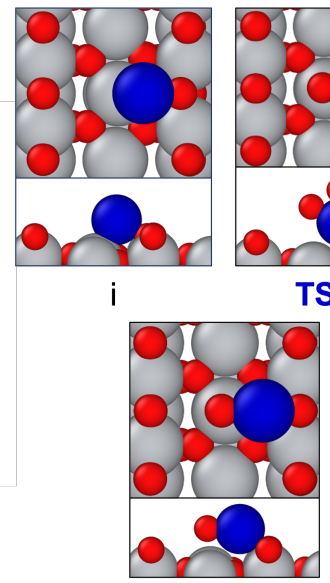

TS2

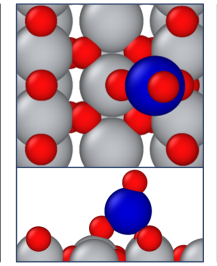

ii

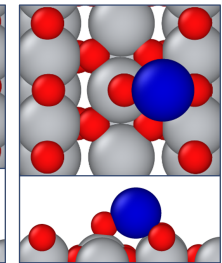

iii

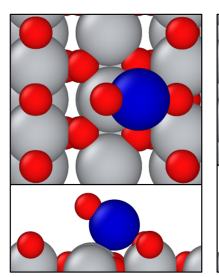

iv

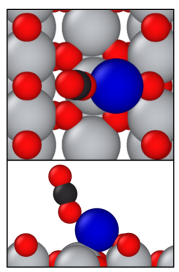

$\mathbf{v}$

Figure S1: ER - Catalytic cycle for ER mechanism. Bold blue states are rate determining intermediate (TDI) and transition state (TDTS) of the mechanism determined via the Energetic Span Model.

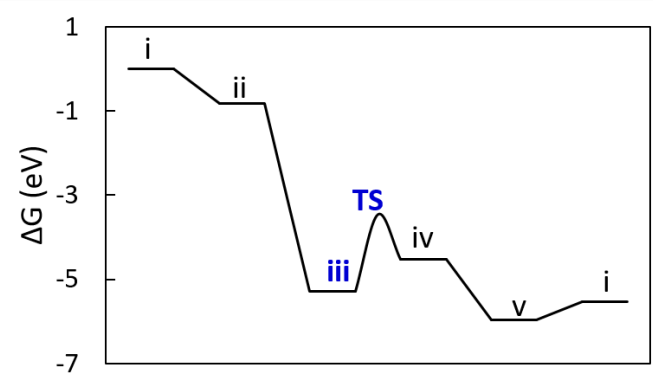

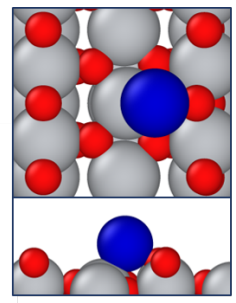

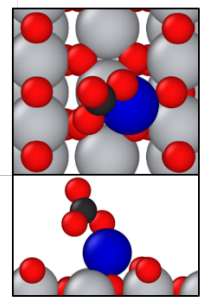

TS

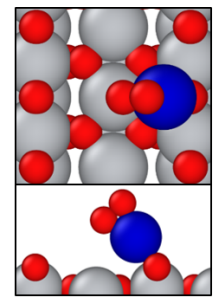

ii

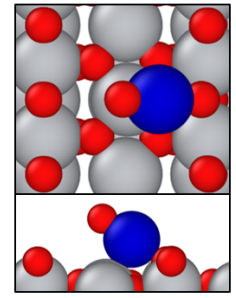

iv

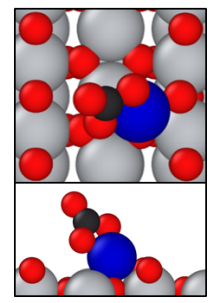

iii

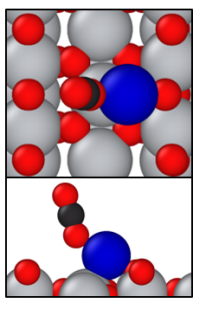

V

Figure S2: $\mathbf{E R}_{\mathbf{C O}_{3}^{\delta-}}-$ Catalytic cycle for ER mechanism with carbonate intermediate.

\section{Proposed Catalytic Cycles for CO Oxidation}

Possible pathways constructed from different $\mathrm{CO}_{2}$ formation mechanisms reported in the Results section of the main text are shown here. Figures S1 - S11 show complete catalytic cycles for which TOFs are calculated using the energetic span model.

\section{Degree of TOF Control}

Degree of turnover frequency control values $\left(X_{T O F}\right)$ of TOF-determining intermediates and transition states of each mechanism are reported in Table S1. Corresponding TDI and TDTS are shown in Table 1 of the main text. The sum of all $X_{T O F}$ for intermediates (transition states) is equal to unity. Therefore, if a single intermediate or transition state limits the rate $X_{T O F}$ is equal to unity. ${ }^{18,19}$

Lower $X_{T O F, T D I}$ and/or $X_{T O F, T D T S}$ values of $\mathbf{E R}+\mathbf{L H}, \mathbf{M v K}_{\mathbf{C O}}$ and $\mathbf{M v K}_{\mathbf{C O}}+\mathbf{E R}$ pathways indicate there are more than two states (one intermediate and one transition state) that limit the rate. The intermediate with co-adsorbed $\mathrm{CO}$ and $\mathrm{O}$ atom (iv in Figure S3) in ER+LH mechanism has the second highest $X_{T O F}$ value of 0.02. For $\mathbf{M v K}_{\mathbf{C O}}, X_{T O F, T D I}$ of step vi in Figure S11 in which two CO molecules are adsorbed on Pt and oxygen 

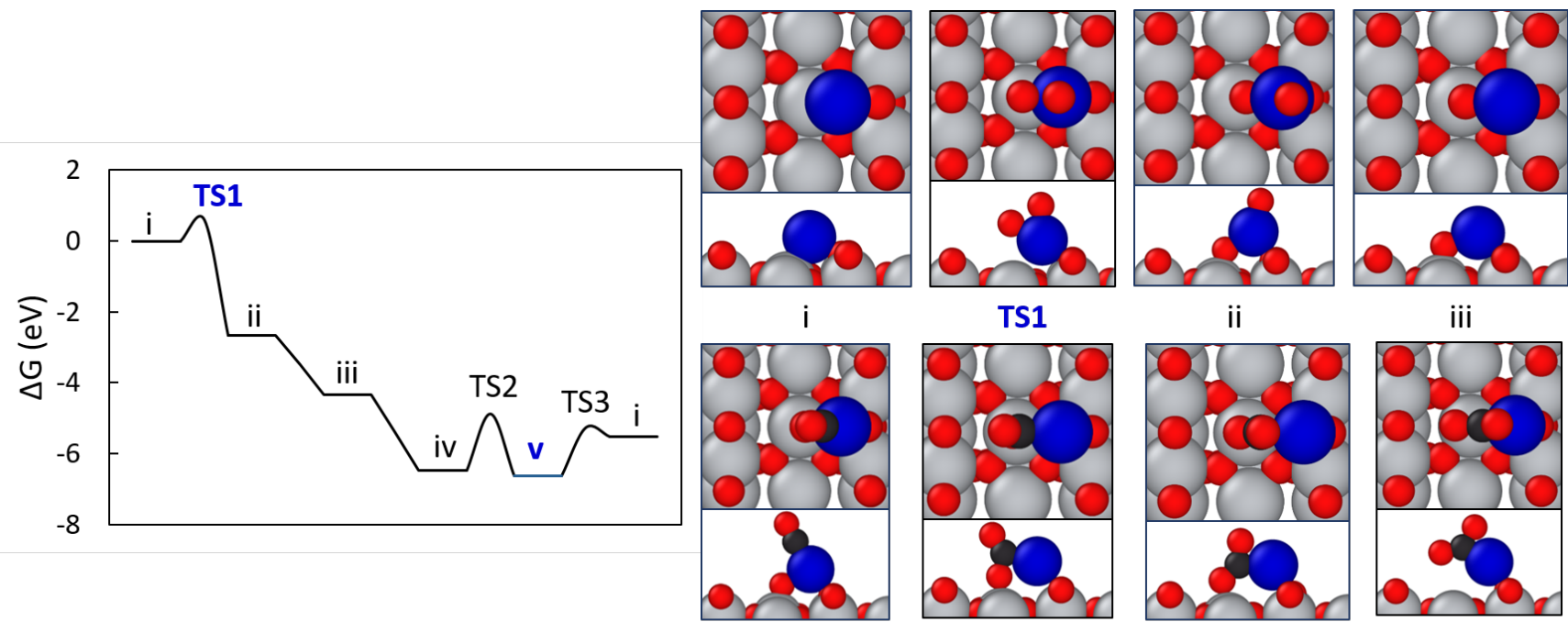

iv

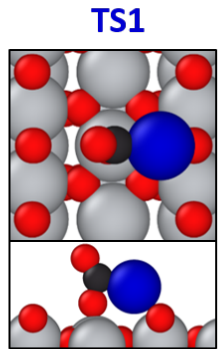

TS2

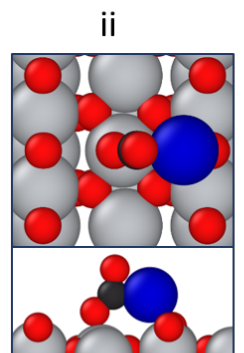

v

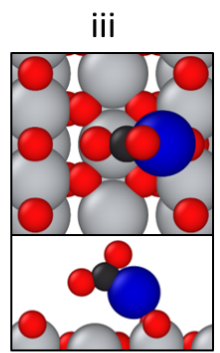

TS3

Figure S3: ER + LH - Catalytic cycle for ER followed by LH mechanism.

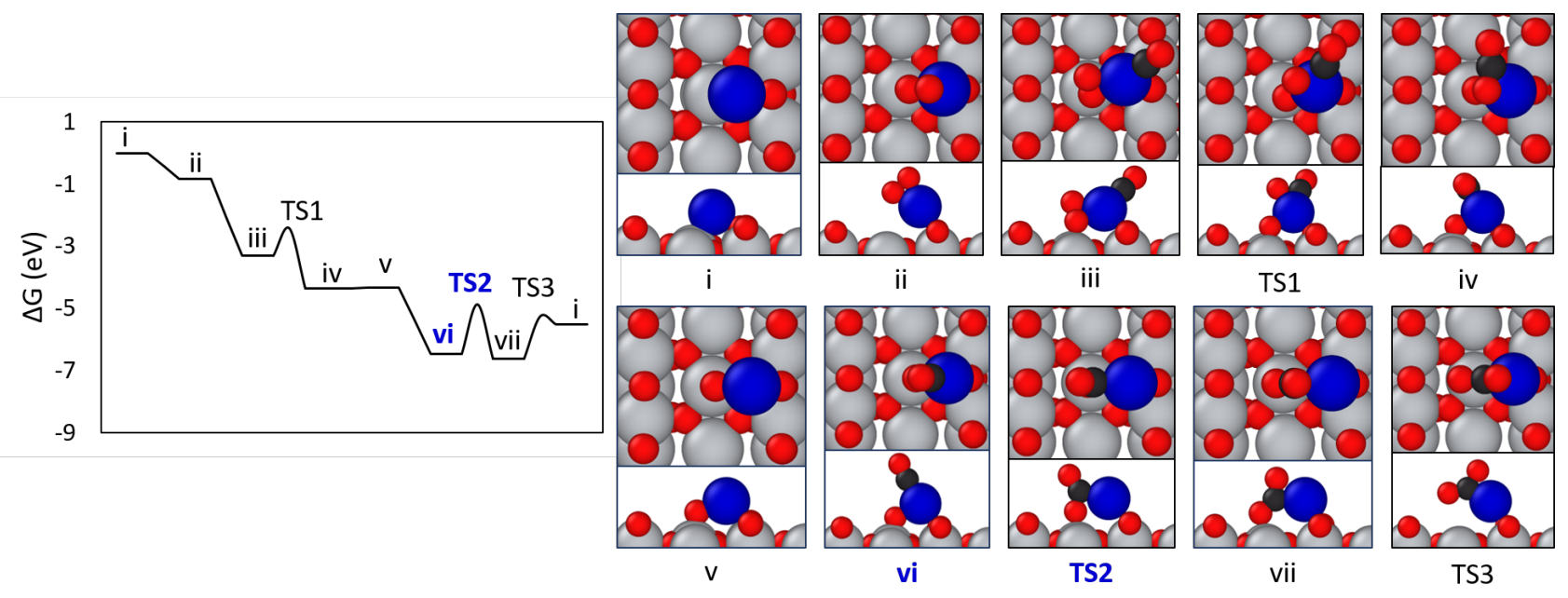

Figure S4: LH - Catalytic cycle for LH mechanism. 

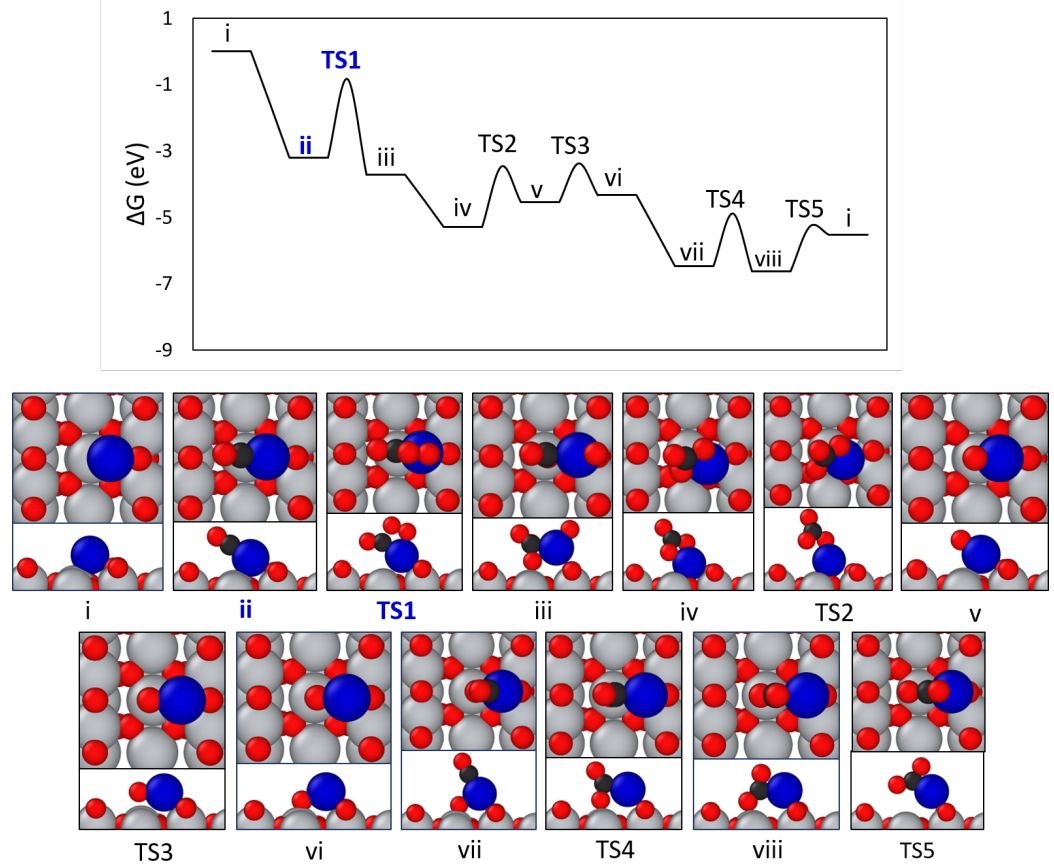

Figure S5: $\mathbf{L H}_{\mathbf{C O}_{3}^{\delta-}}-$ Catalytic cycle for $\mathbf{L H}$ mechanism via the carbonate intermediate.
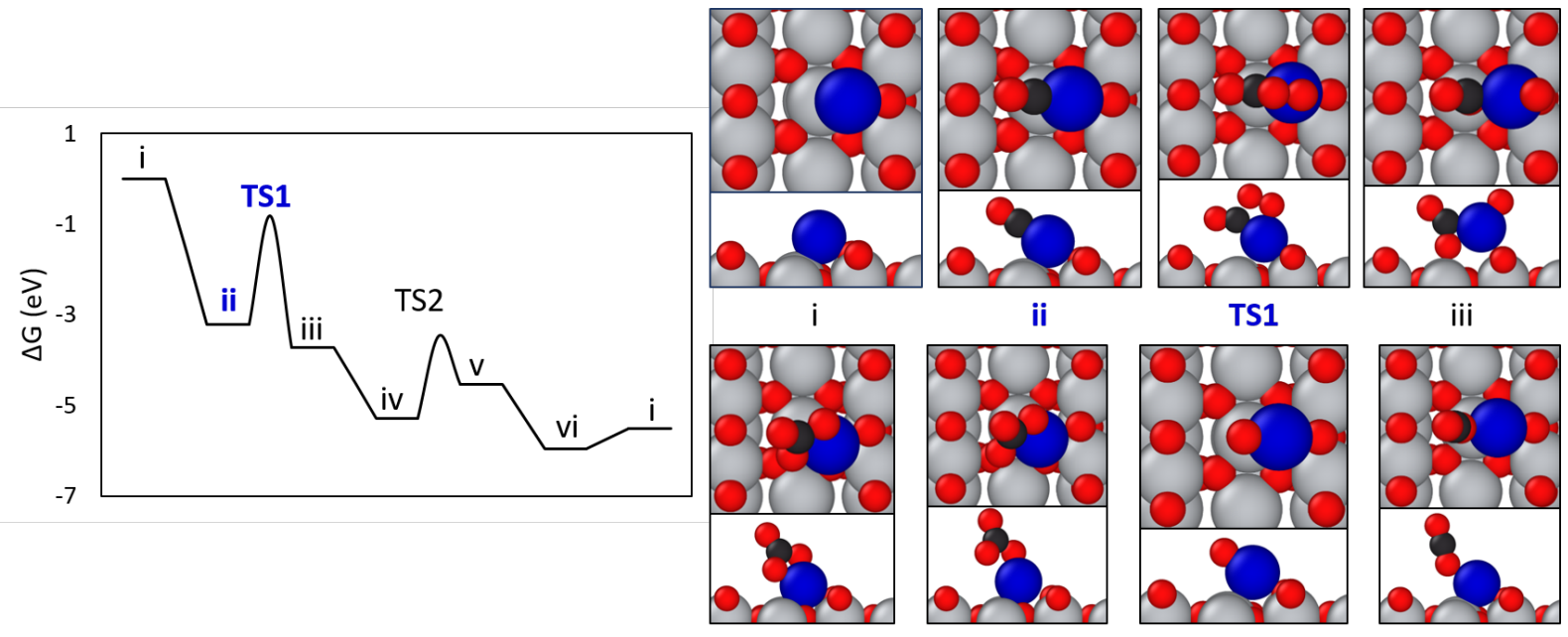

iv

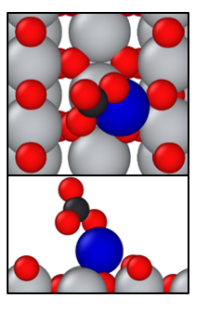

TS2
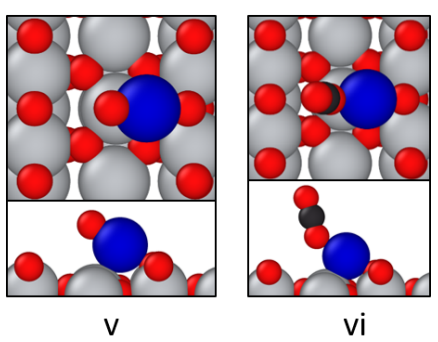

Figure S6: $\mathbf{L H}_{\mathbf{C O}_{3}^{\delta-}} \mathbf{E R}$ - Catalytic cycle for LH followed by ER mechanism via the carbonate intermediate. 

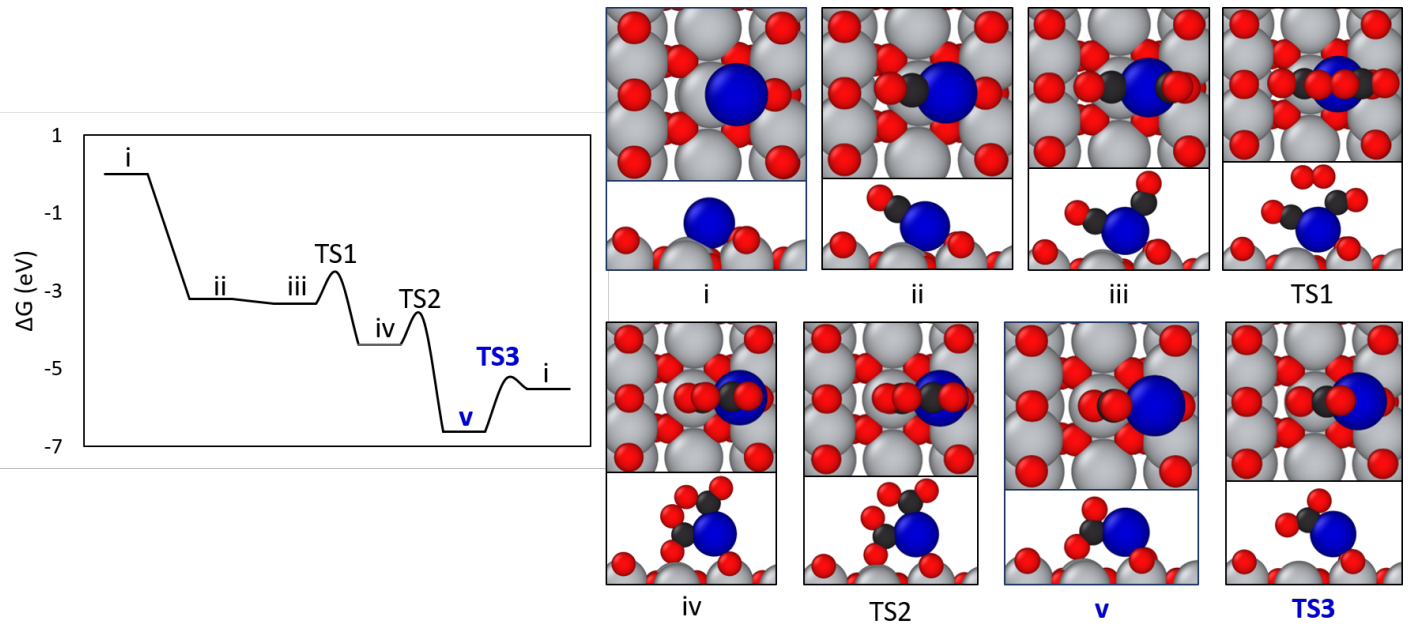

Figure S7: TER - Catalytic cycle for termolecular ER mechanism.
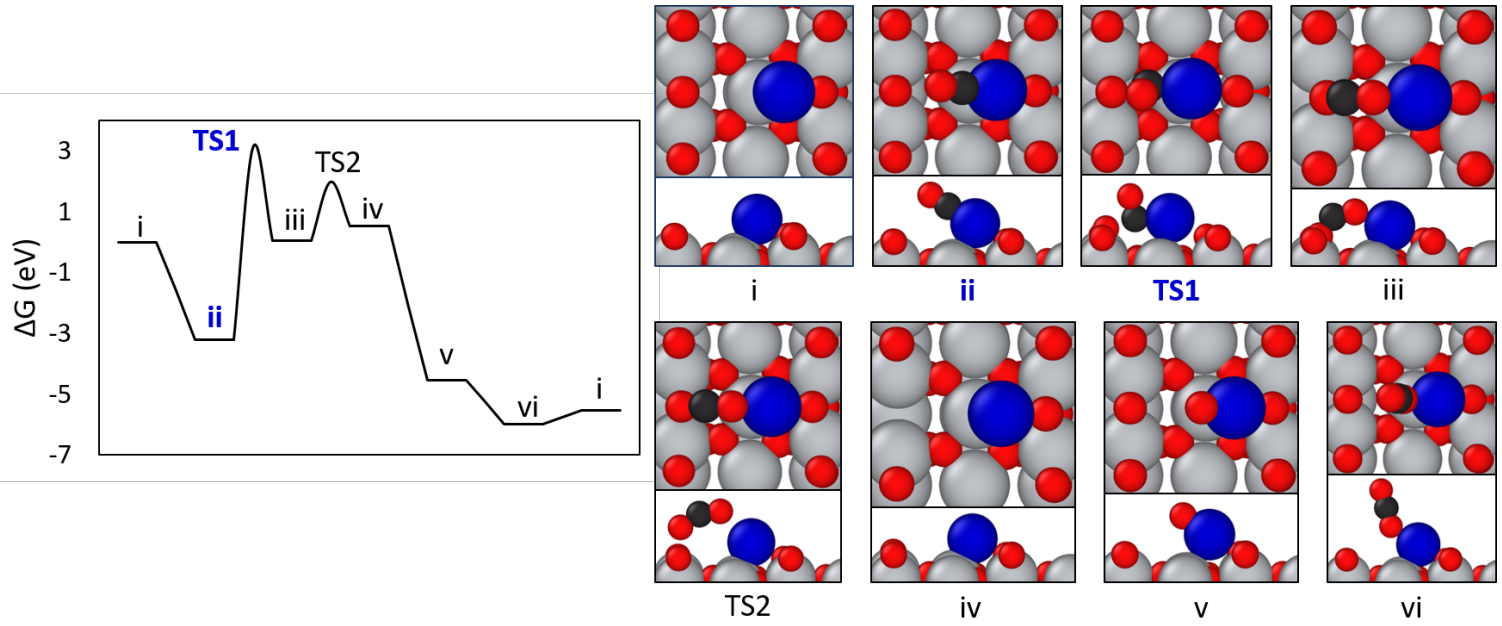

Figure S8: $\mathbf{M v K}_{\mathbf{C O m i g}}$ - Catalytic cycle for MvK mechanism with CO migration.
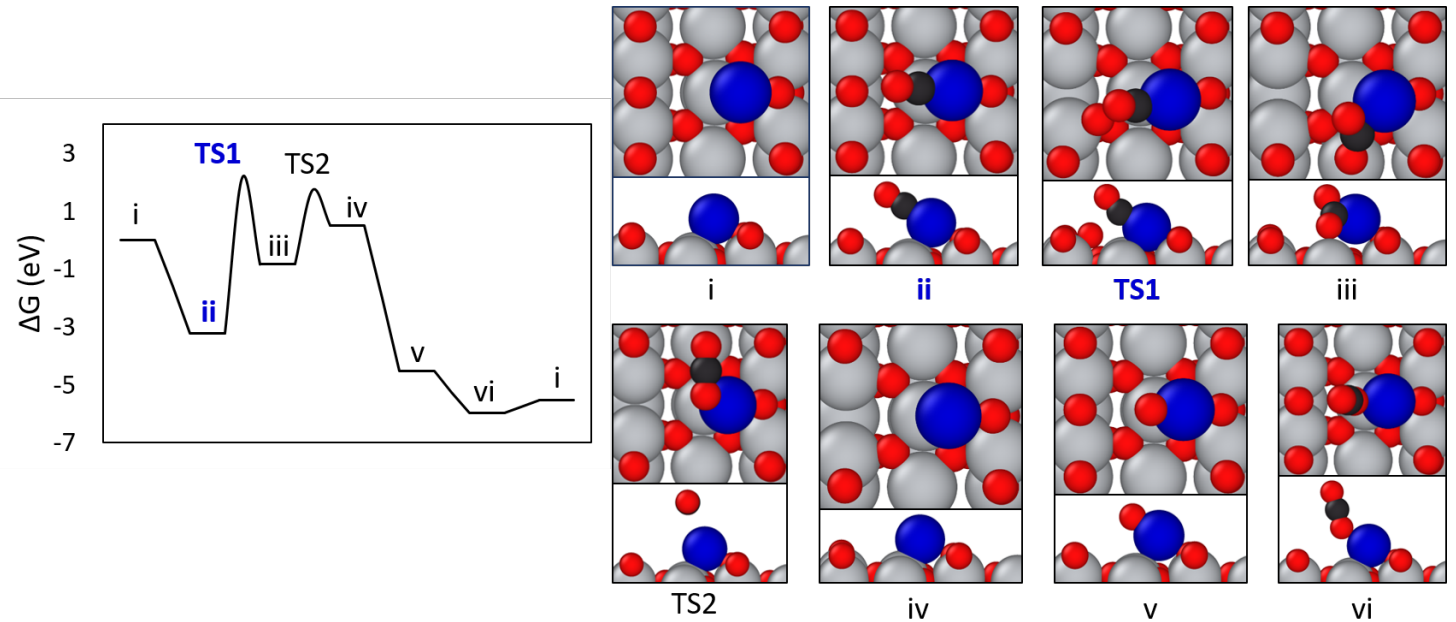

Figure S9: $\mathbf{M v K}_{\text {Omig }}$ - Catalytic cycle for MvK mechanism with lattice O migration. 

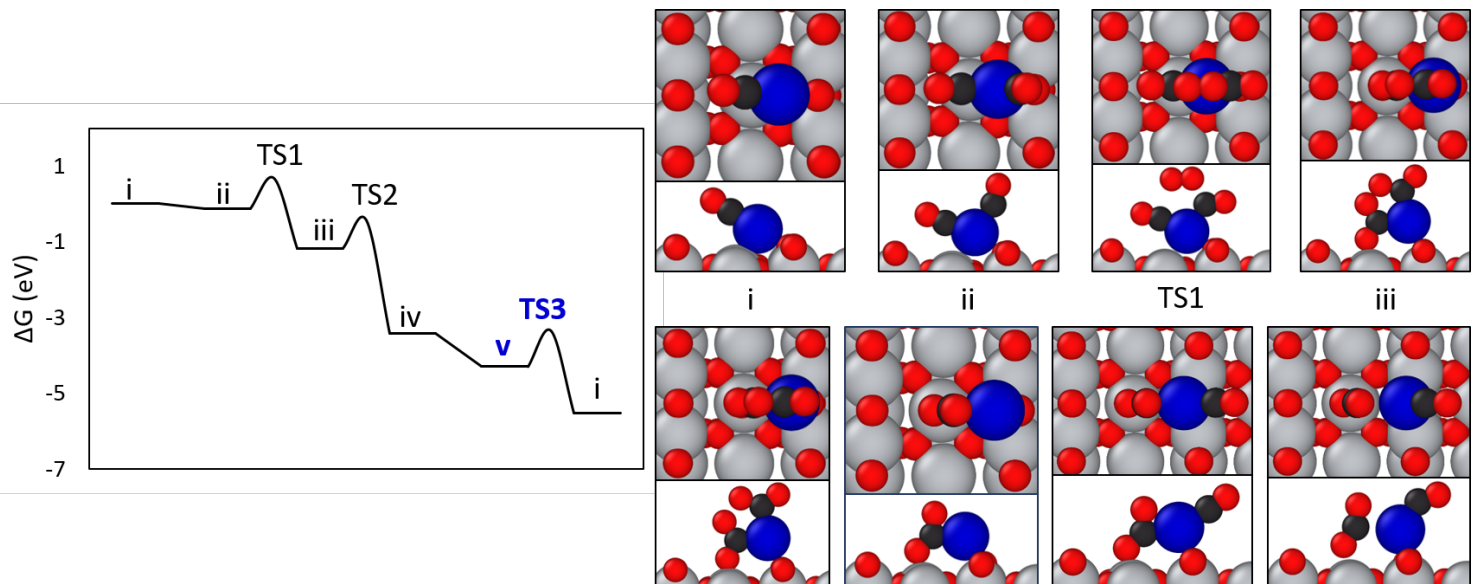

TS2

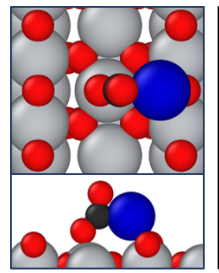

TS1

iii
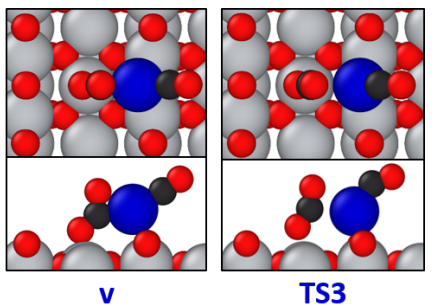

Figure S10: $\mathbf{T E R} \mathbf{C O}_{-}$- Catalytic cycle for TER mechanism with CO-adsorbed $\mathrm{H} 1$ site $\mathrm{Pt}_{1} / \mathrm{TiO}_{2}$ as reference.
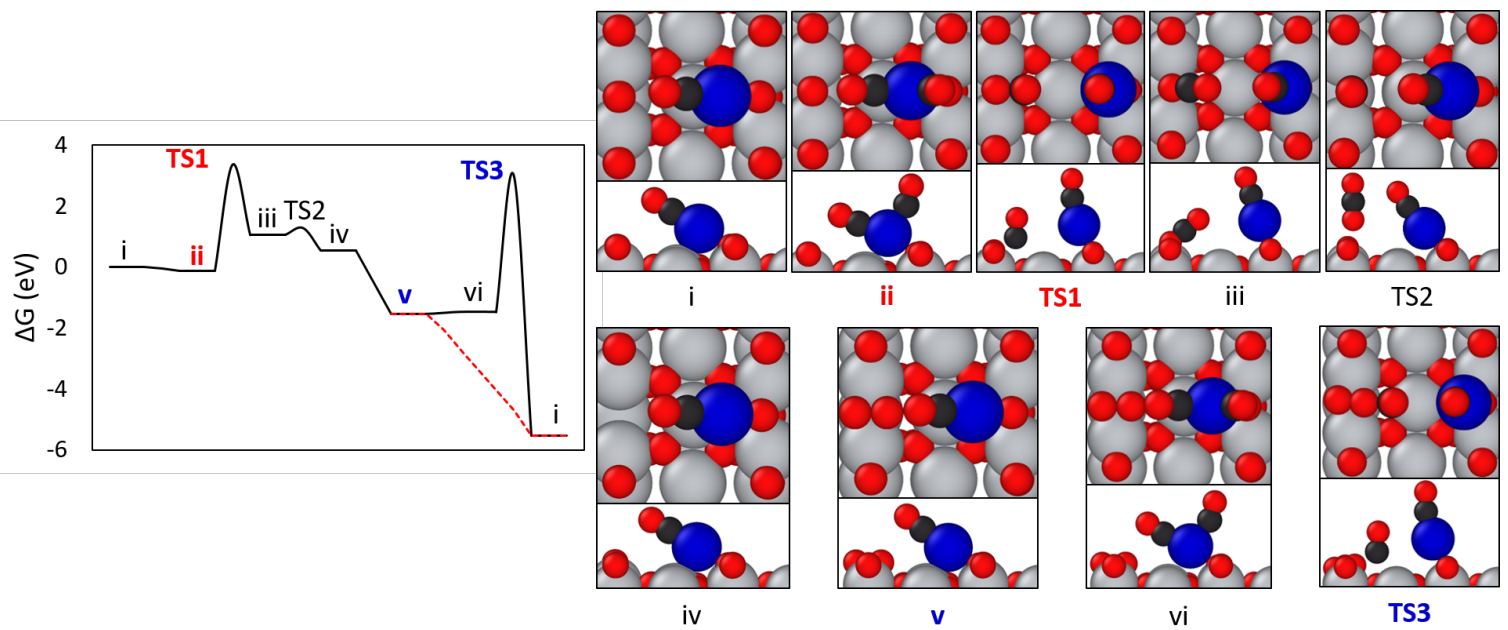

TS3

Figure S11: $\mathbf{M v K} \mathbf{K}_{\mathbf{C O}}$ and $\mathbf{M v K} \mathbf{K}_{\mathbf{C O}}+\mathbf{E R}$ - Catalytic cycles for MvK mechanism for CO-adsorbed $\mathrm{H} 1$ site $\mathrm{Pt}_{1} / \mathrm{TiO}_{2}$ as reference. Bold blue states are TDI and TDTS for $\mathbf{M v K}_{\mathbf{C O}}$. Dashed red line corresponds to direct formation of $\mathrm{CO}_{2}$ from the extra $\mathrm{O}$ on the lattice and gaseous $\mathrm{CO}\left(\mathbf{M v K}_{\mathbf{C O}}+\mathbf{E R}\right)$ and bold red states are corresponding TDI TDTS. 
vacancy is filled with partially dissociated $\mathrm{O}_{2}$ molecule, is 0.06 . Whereas in $\mathbf{M v K} \mathbf{C O}+\mathbf{E R}$, the $\mathrm{CO}$ adsorbed state has the second highest $X_{T O F}$ value. Since this intermediate corresponds to the reference state, $X_{T O F, T D I}$ values are evenly distributed between two steps (states i in Figure S11).

Table S1: Energetic span $(\delta E)$ and degree of TOF control of TDI and TDTS at $298 \mathrm{~K}$ of enumerated low-temperature $\mathrm{CO}$ oxidation pathways. The difference between the free energies of TDTS and TDI $\left(\Delta G=\mathrm{G}_{\mathrm{TDTS}}-\mathrm{G}_{\mathrm{TDI}}\right)$ are also presented.

\begin{tabular}{lcccc}
\hline Pathway & $\begin{array}{c}\delta E \\
(\mathrm{eV})\end{array}$ & $\begin{array}{c}\Delta G \\
(\mathrm{eV})\end{array}$ & $X_{T O F, T D I}$ & $X_{T O F, T D T S}$ \\
\hline \hline $\mathbf{E R}$ & 1.03 & 6.56 & 1.0 & 1.0 \\
$\mathbf{T E R}$ & 1.36 & 1.36 & 1.0 & 1.0 \\
$\mathbf{L H}$ & 1.58 & 1.58 & 1.0 & 1.0 \\
$\mathbf{E R}+\mathbf{L H}$ & 1.69 & 7.21 & 0.98 & 0.98 \\
$\mathbf{E R}_{\mathbf{C O}_{3}^{\delta-}}$ & 1.83 & 1.83 & 1.0 & 1.0 \\
$\mathbf{L H}_{\mathbf{C O}_{3}^{\delta-}}$ & 2.38 & 2.38 & 1.0 & 1.0 \\
$\mathbf{L H}_{\mathbf{C O}} \mathbf{s}_{\mathbf{3}}^{\text {- }}+\mathbf{E R}$ & 2.38 & 2.38 & 1.0 & 1.0 \\
$\mathbf{M v K}_{\text {Omig }}$ & 5.39 & 5.39 & 1.0 & 1.0 \\
$\mathbf{M v K}_{\mathbf{C O m i g}}$ & 6.33 & 6.33 & 1.0 & 1.0 \\
\hline $\mathbf{T E R}_{\mathbf{C O}}$ & 0.95 & 0.95 & 1.0 & 1.0 \\
$\mathbf{M v K}_{\mathbf{C O}}+\mathbf{E R}$ & 3.47 & 3.47 & 0.98 & 1.0 \\
$\mathbf{M v K}_{\mathbf{C O}}$ & 4.55 & 4.55 & 0.94 & 1.0 \\
\hline
\end{tabular}

\section{Partial Pressure Effects on TOF Analysis}

Gibbs free energies of gas molecules are modified by addition of the partial free energies of each species using the following equation: ${ }^{20}$

$$
G=E_{D F T}+E_{Z P E}+U-T S+k_{B} T \ln \left(P_{i} / P^{0}\right)
$$

where $\mathrm{k}_{\mathrm{B}}$ is the Boltzmann constant, $\mathrm{T}$ is temperature, $\mathrm{P}$ is the partial pressure of the species and $\mathrm{P}^{0}$ is the standard pressure.

To model pressure effects, a total pressure of 1 bar is selected with CO partial pressure of 0.01 bar at the inlet and $\mathrm{O}_{2} / \mathrm{CO}$ feed ratio of $4 .{ }^{21}$ Ten percent of the $\mathrm{CO}$ is assumed to convert to $\mathrm{CO}_{2}$, resulting in partial pressures of $\mathrm{CO}$, $\mathrm{O}_{2}$ and $\mathrm{CO}_{2}$ of $0.009,0.038,0.001$ bar, respectively.

The following equation can be used to calculate TOF upon inclusion of partial pressures of gas molecules: ${ }^{22}$

$$
\mathrm{TOF}_{\mathrm{P}} \approx T O F \prod_{h} \frac{\delta R_{h, x, y}}{\delta P_{h, x, y}}
$$

where TOF is calculated using Eq (1) in the main manuscript with Gibbs free energies including partial pressures of reactants and products. $\mathrm{x}$ and y are labels for TDTS and TDI, respectively. $\delta R_{h, x, y}$ and $\delta P_{h, x, y}$ are either equal to 1 or partial pressure of reactants (products) $\left[R_{h}\right]\left(\left[P_{h}\right]\right)$ depending on the catalytic cycle:

$$
\begin{aligned}
\delta R_{h, i, j} & = \begin{cases}{\left[R_{h}\right],} & \text { if } p_{h}(i)>p_{h}(j) \\
1, & \text { otherwise }\end{cases} \\
\delta P_{h, i, j} & = \begin{cases}{\left[P_{h}\right],} & \text { if } p_{h}(i)<p_{h}(j) \\
1, & \text { otherwise }\end{cases}
\end{aligned}
$$

where $p_{h}(i)$ and $p_{h}(j)$ are cyclic permutations:

$$
p_{h}(i)= \begin{cases}i-h+1, & \text { if } i=h, h+1, \ldots, N \\ i-h+1+N, & \text { if } i=1,2, \ldots, h-1\end{cases}
$$


$\mathrm{N}$ is the number of intermediates in the catalytic cycle.

According to the energetic span approximation, partial pressure of reactants enter in between TDI and TDTS increase the TOF, whereas partial pressure of products that leave the system between TDI and TDTS hinder the rate. Partial pressures of the remaining species that are involved in the reaction are negligible. Using the TOF values calculated, we obtain Table S2 by including the partial pressures of gas molecules.

Table S2: Room temperature turnovers calculated with partial pressure dependent $\left(\mathrm{TOF}_{P}\right)$ and independent $(\mathrm{TOF})$ free energies. Reactants that enter and products leave the reaction cycle between TDI and TDTS are also reported for each pathway.

\begin{tabular}{lcccc}
\hline Pathway & $\begin{array}{c}\text { Reactants } \\
\text { Enter }\end{array}$ & $\begin{array}{c}\text { Products } \\
\text { Leave }\end{array}$ & $\begin{array}{c}\text { TOF } \\
\left(\mathrm{h}^{-1}\right)\end{array}$ & $\begin{array}{c}\mathrm{TOF}_{\mathrm{P}} \\
\left(\mathrm{h}^{-1}\right)\end{array}$ \\
\hline \hline $\mathbf{E R}$ & $\mathrm{O}_{2}$ & - & $8.50 \times 10^{-2}$ & $1.22 \times 10^{-1}$ \\
$\mathbf{T E R}$ & - & - & $2.51 \times 10^{-7}$ & $2.51 \times 10^{-7}$ \\
$\mathbf{L H}$ & - & - & $3.98 \times 10^{-11}$ & $3.98 \times 10^{-11}$ \\
$\mathbf{E R + \mathbf { H }}$ & $\mathrm{O}_{2}$ & $\mathrm{CO}_{2}$ & $6.73 \times 10^{-13}$ & $5.98 \times 10^{-10}$ \\
$\mathbf{E R}_{\mathbf{C O}_{3}^{\delta-}}$ & - & - & $2.27 \times 10^{-15}$ & $2.27 \times 10^{-15}$ \\
$\mathbf{L H}_{\mathbf{C O}_{3}^{\delta-}}$ & $\mathrm{O}_{2}$ & - & $1.09 \times 10^{-24}$ & $1.57 \times 10^{-27}$ \\
$\mathbf{L H}_{\mathbf{C O}_{3}^{\delta-}+\mathbf{E R}}$ & $\mathrm{O}_{2}$ & - & $1.09 \times 10^{-24}$ & $1.57 \times 10^{-27}$ \\
$\mathbf{M v K}_{\mathbf{O m i g}}$ & - & - & $1.30 \times 10^{-75}$ & $1.30 \times 10^{-75}$ \\
$\mathbf{M v K}_{\mathbf{C O m i g}}$ & - & - & $1.62 \times 10^{-91}$ & $1.62 \times 10^{-91}$ \\
\hline $\mathbf{T E R} \mathbf{C O}$ & - & - & 1.72 & 1.71 \\
$\mathbf{M v K}_{\mathbf{C O}}+\mathbf{E R}$ & - & - & $4.19 \times 10^{-43}$ & $1.33 \times 10^{-43}$ \\
$\mathbf{M v K}_{\mathbf{C O}}$ & $\mathrm{CO}$ & - & $2.48 \times 10^{-61}$ & $2.16 \times 10^{-65}$ \\
\hline
\end{tabular}

\section{Pt Charges During CO Oxidation Reaction}

Table S3 shows the evolution of Pt charges along the reaction pathways shown in Figures S1-S11. The only negative charge $(-0.10 \mathrm{e})$ is when there is a vacancy on the surface and no additional adsorbates are present ( $\mathbf{M v K}_{\mathbf{O m i g}}$ and $\mathbf{M v K}_{\mathbf{C O m i g}}$ - step iv). Positive Pt charge ranges between $+0.08 \mathrm{e}$ to $+1.25 \mathrm{e}$, the less positive corresponds to the geometry where one $\mathrm{CO}$ attached to $\mathrm{O}_{b r}$ and one $\mathrm{CO}$ adsorbed on $\mathrm{Pt}(\mathbf{M v K} \mathbf{C O}$ - step iii) whereas the most positive $\mathrm{Pt}$ is when $\mathrm{O}_{2}$ is dissociatively adsorbed on $\mathrm{Pt}$ in the second step of ER mechanism.

Table S3: Pt charges (e) on the intermediates in enumerated reaction pathways

\begin{tabular}{lllllllll}
\hline Pathway & step 1 & step 2 & step 3 & step 4 & step 5 & step 6 & step 7 & step 8 \\
\hline ER & +0.12 & +1.25 & +0.74 & +0.50 & +0.17 & & & \\
ER+LH & +0.12 & +1.25 & +0.74 & +0.84 & +0.44 & & & \\
ER $_{\mathbf{C O}_{\mathbf{3}}^{\delta-}}$ & +0.12 & +0.68 & +0.80 & +0.50 & +0.17 & & & \\
$\mathbf{L H}^{\mathbf{L}}$ & +0.12 & +0.68 & +0.81 & +0.92 & +0.74 & +0.84 & +0.44 & \\
$\mathbf{L H}_{\mathbf{3}}^{\delta-}$ & +0.12 & +0.13 & +0.89 & +0.80 & +0.50 & +0.74 & +0.84 & +0.44 \\
$\mathbf{T E O}_{\mathbf{3}}+\mathbf{E R}$ & +0.12 & +0.13 & +0.89 & +0.80 & +0.50 & +0.17 & & \\
$\mathbf{M v K}_{\mathbf{C O m i g}}$ & +0.12 & +0.13 & +0.23 & +0.45 & +0.44 & & & \\
$\mathbf{M v K}_{\mathbf{O m i g}}$ & +0.12 & +0.13 & +0.24 & -0.10 & +0.50 & & & \\
$\mathbf{T E R}_{\mathbf{C O}}$ & +0.13 & +0.23 & +0.45 & +0.44 & +0.57 & & & \\
$\mathbf{M v K}_{\mathbf{C O}}$ & +0.13 & +0.23 & +0.08 & +0.14 & +0.13 & +0.22 & & \\
$\mathbf{M v K}_{\mathbf{C O}}+\mathbf{E R}$ & +0.13 & +0.23 & +0.08 & +0.14 & +0.13 & & & \\
\hline
\end{tabular}

\section{Properties of $\mathrm{H} 1$ Site and Gas Molecules}

The strongest Pt-binding site on vacancy-free rutile $\mathrm{TiO}_{2}(110)$ surface, namely the hollow $\mathrm{H} 1$ (step i in Figure $\mathrm{S} 1$ ), is selected as the representative site for this study. ${ }^{23}$ The $\mathrm{Pt}$ atom is coordinated to one bridging $\mathrm{O}$ (1.923 $\AA$ ) and 
one five-coordinated Ti atom $(2.453 \AA)$. There are two basal oxygen atoms close to Pt at a larger distance of $2.851 \AA$.

Geometry optimization and vibrational analysis of gas molecules that participate in $\mathrm{CO}$ oxidation reaction, namely $\mathrm{CO}, \mathrm{O}_{2}$, and $\mathrm{CO}_{2}$ are performed with the same unit cell used in the rest of the calculations. Distance between corresponding atoms on each molecule, Bader charges on atoms and energies of the molecules are reported in Table S4. Zero point energies (ZPE) are obtained from vibrational analyses and free energies are calculated at room temperature considering the contributions from translations, rotations and vibrations.

Table S4: Electronic, zero point and free energies, Bader charges and atomic distances of gas molecules. Free energies are calculated at room temperature.

\begin{tabular}{lrrr} 
& $\mathrm{CO}$ & $\mathrm{O}_{2}$ & $\mathrm{CO}_{2}$ \\
\hline Electronic Energy $(\mathrm{eV})$ & -16.88 & -12.31 & -26.22 \\
$\mathrm{ZPE}(\mathrm{eV})$ & 0.14 & 0.10 & 0.32 \\
Free Energy $(\mathrm{eV})$ & -17.36 & -12.87 & -26.55 \\
Bader Charge on C (e) & +1.12 & - & +2.17 \\
Bader Charge on O (e) & -1.12 & 0 & -1.08 \\
Distance $(\AA)$ & 1.133 & 1.226 & 1.165 \\
\hline
\end{tabular}

\section{Binding Energies of Adsorbates on Pt}

Table S5 shows the binding energies of adsorbates on Pt atom calculated from electronic $(\Delta E)$ and free energies $(\Delta G)$. The reference state consists of isolated catalyst and gas phase molecules.

Table S5: Binding energies of molecules on Pt atom calculated via potential and free energies.

\begin{tabular}{|c|c|c|c|c|c|c|c|}
\hline & Linear $\mathrm{CO}_{2}$ & Bent $\mathrm{CO}_{2}$ & Two $\mathrm{CO}_{2}$ & $\mathrm{CO}$ & Two CO & $\mathrm{O}_{2}$ & Dissociative $\mathrm{O}_{2}$ \\
\hline$\Delta E(\mathrm{eV})$ & -1.01 & -1.74 & -0.09 & -3.88 & -4.57 & -1.45 & -3.32 \\
\hline$\Delta G(\mathrm{eV})$ & -0.44 & -1.10 & +1.14 & -3.21 & -3.33 & -0.83 & -2.67 \\
\hline
\end{tabular}




\section{References}

[1] Kresse, G.; Hafner, J. Ab initio molecular dynamics for liquid metals. Physical Review B 1993, 47, 558.

[2] Kresse, G.; Hafner, J. Ab initio molecular-dynamics simulation of the liquid-metal-amorphous-semiconductor transition in germanium. Physical Review B 1994, 49, 14251.

[3] Kresse, G.; Furthmüller, J. Efficiency of ab-initio total energy calculations for metals and semiconductors using a plane-wave basis set. Computational Materials Science 1996, 6, 15-50.

[4] Kresse, G.; Furthmüller, J. Efficient iterative schemes for ab initio total-energy calculations using a plane-wave basis set. Physical Review B 1996, 54, 11169.

[5] Sun, J.; Ruzsinszky, A.; Perdew, J. P. Strongly constrained and appropriately normed semilocal density functional. Physical Review Letters 2015, 115, 036402.

[6] Larsen, A. H.; Mortensen, J. J.; Blomqvist, J.; Castelli, I. E.; Christensen, R.; Dułak, M.; Friis, J.; Groves, M. N.; Hammer, B.; Hargus, C.; Hermes, E. D.; Jennings, P. C.; Jensen, P. B.; Kermode, J.; Kitchin, J. R.; Kolsbjerg, E. L.; Kubal, J. K.; Kaasbjerg, K.; Steen, L.; Maronsson, J. B.; Maxson, T.; Olsen, T.; Pastewka, L.; Peterson, A.; Rostgaard, C.; Schiøtz, J.; Schütt, O.; Strange, M.; Thygesen, K. S.; Vegge, T.; Vilhelmsen, L.; Walter, M.; Zeng, Z.; Jacobsen, K. W. The atomic simulation environment-a Python library for working with atoms. Journal of Physics: Condensed Matter 2017, 29, 273002.

[7] Blöchl, P. E. Projector augmented-wave method. Physical review B 1994, 50, 17953.

[8] Kresse, G.; Joubert, D. From ultrasoft pseudopotentials to the projector augmented-wave method. Physical Review B 1999, 59, 1758.

[9] Tang, W.; Sanville, E.; Henkelman, G. A grid-based Bader analysis algorithm without lattice bias. Journal of Physics: Condensed Matter 2009, 21, 084204.

[10] Yu, M.; Trinkle, D. R. Accurate and efficient algorithm for Bader charge integration. The Journal of Chemical Physics 2011, 134, 064111.

[11] Mills, G.; Jónsson, H. Quantum and thermal effects in $\mathrm{H}_{2}$ dissociative adsorption: Evaluation of free energy barriers in multidimensional quantum systems. Physical Review Letters 1994, 72, 1124.

[12] Henkelman, G.; Jónsson, H. Improved tangent estimate in the nudged elastic band method for finding minimum energy paths and saddle points. The Journal of Chemical Physics 2000, 113, 9978-9985.

[13] Henkelman, G.; Uberuaga, B. P.; Jónsson, H. A climbing image nudged elastic band method for finding saddle points and minimum energy paths. The Journal of Chemical Physics 2000, 113, 9901-9904.

[14] Frederiksen, T.; Paulsson, M.; Brandbyge, M.; Jauho, A.-P. Inelastic transport theory from first principles: Methodology and application to nanoscale devices. Physical Review B 2007, 75, 205413.

[15] Nørskov, J. K.; Studt, F.; Abild-Pedersen, F.; Bligaard, T. Fundamental concepts in heterogeneous catalysis; John Wiley \& Sons, 2014; pp 31-34.

[16] Sandler, S. I. An Introduction to Applied Statistical Thermodynamics; John Wiley \& Sons, 2010; pp 44-58.

[17] Ammal, S. C.; Heyden, A. Titania-Supported Single-Atom Platinum Catalyst for Water-Gas Shift Reaction. Chemie Ingenieur Technik 2017, 89, 1343-1349.

[18] Kozuch, S.; Shaik, S. A combined kinetic- quantum mechanical model for assessment of catalytic cycles: Application to cross-coupling and Heck reactions. Journal of the American Chemical Society 2006, 128, 33553365 .

[19] Kozuch, S.; Shaik, S. How to conceptualize catalytic cycles? The energetic span model. Accounts of Chemical Research 2011, 44, 101-110.

[20] Ammal, S. C.; Heyden, A. Water-gas shift activity of atomically dispersed cationic platinum versus metallic platinum clusters on titania supports. ACS Catalysis 2017, 7, 301-309. 
[21] Lou, Y.; Liu, J. CO oxidation on metal oxide supported single Pt atoms: the role of the support. Industrial \& Engineering Chemistry Research 2017, 56, 6916-6925.

[22] Kozuch, S.; Shaik, S. Kinetic-quantum chemical model for catalytic cycles: the Haber- Bosch process and the effect of reagent concentration. The Journal of Physical Chemistry A 2008, 112, 6032-6041.

[23] Humphrey, N.; Bac, S.; Mallikarjun Sharada, S. Ab Initio Molecular Dynamics Reveals New Metal-Binding Sites in Atomically Dispersed $\mathrm{Pt}_{1} / \mathrm{TiO}_{2}$ Catalysts. The Journal of Physical Chemistry C 2020, 124, 24187-24195. 